\title{
Análisis del diseño aerodinámico de un alerón preparado para competencia utilizando simulaciones numéricas de dinámica de fluidos computacional (DFC)
}

\author{
Giovanni F. Samaniego', Byron Guerrero² y Jaime F. Antamba ${ }^{1 *}$ \\ (1) Facultad de Ciencias Técnicas, Escuela de Ingeniería Automotriz, UIDE, Casilla 170411, Quito - Ecuador. \\ (correo-e: gisamaniegofl@uide.edu.ec; jferantamba@gmail.com) \\ (2) School of Mechanical Engineering, Faculty of Engineering Mathematics and Computer Science, The University of \\ Adelaide, Adelaide 5000, SA., Australia. (correo-e: byron.guerrerohinojosa@adelaide.edu.au) \\ * Autor a quien debe ser dirigida la correspondencia.
}

Recibido Ago. 26, 2020; Aceptado Oct. 23, 2020; Versión final Dic. 1, 2020, Publicado Abr. 2021

\begin{abstract}
Resumen
La presente investigación analiza numéricamente el comportamiento aerodinámico de vehículo Suzuki Forsa con un alerón para competencia en pista. Para el diseño del alerón, se realiza una simulación en ANSYS Fluent utilizando el modelo de turbulencia RANS K- $\epsilon$ con una malla gruesa y una malla refinada. La malla refinada permitió validar los datos considerando una distancia de pared adimensional $\left(\mathrm{y}^{+}=5\right)$ y una tasa de inflación de 1,20. Con esta configuración, se realizaron simulaciones de dinámica de fluidos computacional con distintos ángulos (5ㅜ, 10ำ $15^{\circ}$ y $\left.20^{\circ}\right)$ con un tiempo de simulación de 10 horas para cada ángulo propuesto. Los resultados muestran que un ángulo de 20 을 brinda una fuerza de sustentación negativa (fuerza aerodinámica) adecuada para que los neumáticos tengan un mejor agarre con la superficie de rodadura y alcanzar la mejor eficiencia aerodinámica. Se concluye que la configuración de alerón con ángulo de incidencia de $20^{\circ}$ brinda la mayor carga aerodinámica con la mejor eficiencia aerodinámica para el vehículo Suzuki Forsa.
\end{abstract}

Palabras clave: simulación; aerodinámica; sustentación; arrastre

\section{Aerodynamic design analysis of a racing airfoil by computational fluid dynamic (CFD) numerical simulations}

\begin{abstract}
The present research study examines the aerodynamic behavior of a Suzuki Forsa vehicle with a racing airfoil. The ANSYS Fluent software and the RANS K- $\epsilon$ turbulence model are used to perform a numerical simulation. A coarse and a refined mesh are used to design the airfoil. The data is validated with the refined mesh by using a normalized wall distance $\left(\mathrm{y}^{+}=5\right)$ and an inflation rate of 1.20 . This configuration is used to perform computational fluid dynamics simulations with different angles of attack $\left(5^{\circ}, 10^{\circ}, 15^{\circ}\right.$, and $\left.20^{\circ}\right)$. The simulation time was 10 hours for the proposed angles of attack. The results show that the $20^{\circ}$ angle of attack provides an adequate negative sustentation force (aerodynamic force) to improve tire grip with tread surface and achieve the most efficient aerodynamic. It is concluded that the $20^{\circ}$ angle of attack configuration provides the most downforce and the most efficient aerodynamic configuration for Suzuki Forsa vehicles.
\end{abstract}




\section{INTRODUCCIÓN}

La dinámica de fluidos computacional (CFD) se ha convertido en uno de las principales herramientas de diseño para la aerodinámica externa de los vehículos actuales, debido a la facilidad de obtener hardware y software sofisticados para el manejo de cualquier tipo de geometrías, siendo la geometría de las carrocerías de los automóviles una de las más complejas (Aston y Revell, 2015). Los diferentes flujos de aire se estudian usualmente resolviendo las ecuaciones de Navier-Stokes mediante métodos promediados (RANS) o la simulación de grandes remolinos (LES). La simulación LES genera resultados de alta precisión. Por ello, este método se ha vuelto popular en la industria aeroespacial (Heinz, 2020).

Para las configuraciones automotrices típicas, existe separación (turbulencia) detrás de la carrocería del automóvil, lo que tiene un gran impacto en la resistencia y la eficiencia del vehículo. Si bien hay otras áreas importantes alrededor y dentro del automóvil, la capacidad de una simulación CFD para capturar esta región, donde existen movimientos caóticos de las partículas del fluido, es en gran parte una función de la capacidad predictiva de los modelos de turbulencia. Debido a la competencia dentro de la industria automotriz los resultados de las investigaciones en estos campos son confidenciales (Kim y Han, 2016). Actualmente, las simulaciones numéricas de capa límite son comparables con los experimentos. De hecho, no existe duda de que las simulaciones numéricas de las Ecuaciones de Navier-Stokes son sólo una clase diferente de experimento. Por lo tanto, no existe razón para esperar que una simulación numérica sea menos precisa que un experimento (Jimenez, 2011). Además, las simulaciones numéricas de flujo de fluidos tienen la ventaja de que el flujo puede ser analizado en cualquier punto.

En muchos casos, se ha demostrado que técnicas de simulación numérica directa (DNS) de las ecuaciones de gobierno generan resultados más confiables que las experimentales, pues se evita el ruido y las desviaciones propias de la instrumentación requerida para llevar a cabo los experimentos. De hecho, utilizar los túneles de viento para el diseño automotriz puede presentar desventajas. Por ejemplo, los vehículos de grandes longitudes pueden perturbar el flujo, o el movimiento relativo entre el vehículo, la carretera y la rotación de los neumáticos resulta muy difícil de reproducir en una facilidad experimental (Hucho y Sovran, 1993; Johansson y Katz, 2002). En general, es aceptado que las herramientas CFD proporcionan suficiente precisión para respaldar el desarrollo aerodinámico. Como resultado, casi todos los fabricantes de automóviles de hoy hacen un uso significativo de CFD para el diseño y optimización de formas de vehículos (Wang at al, 2014).

Para el diseño aerodinámico y el análisis de capa límite, el modelo de turbulencia RANS $k-\epsilon$, es uno de los más populares tomando en cuenta que este requiere de menor poder computacional comparado con otras técnicas de simulación tales como los modelos LES y la simulación numérica directa (DNS) (Kobayashi y Kitoh, 1992). De hecho, se ha demostrado que el modelo $k-\epsilon$, genera aproximaciones satisfactorias en la predicción de las fuerzas de arrastre, siempre y cuando sus coeficientes estén calibrados adecuadamente (Wang y Yang ,2015). Adicionalmente, se puede mencionar, que en general las técnicas CFD proveen de una herramienta poderosa para determinar las formas y las características aerodinámicas en vehículos (Dhaubhadel, 1996). De hecho, en la actualidad la simulación CFD se ha convertido en una herramienta irremplazable para el diseño de álabes aerodinámicos, pues esta técnica provee de datos detallados en la distribución del campo de presión y de arrastre (Katz, 2006).

La configuración adecuada de los parámetros de la simulación CFD para vehículos es crítica para obtener resultados confiables, algunos investigadores, han desarrollado modelos de automóviles genéricos, como el modelo SAE y el modelo Ahmed (Heft at al, 2012), facilitándose la relación de los fenómenos observados con áreas específicas y así comprender el flujo básico en las estructuras analizadas. La distancia del modelo a la entrada y a la salida es de $3 \mathrm{~L}$ y $6 \mathrm{~L}$, respectivamente, siendo $\mathrm{L}$ la longitud del vehículo (Sterken at al, 2016). La tasa de inflación debe ser configurada al $20 \%$ con una cantidad de capas prismáticas en la capa límite de 5 y un factor de relación de 0.50 (Lanfrit, 2005). Las distribuciones del valor de distancia de pared adimensional $y^{+}$en las superficies del modelo en este estudio son menores a 5 en las áreas de separación y de 10 a 15 en el resto del modelo superficies, demostrando una buena resolución cerca de la pared, especialmente en zonas de separación (Altinisik at al, 2015).

Las funciones de pared escalable son seleccionadas para el modelo RANS $k-\epsilon$ con la finalidad de obtener resultados consistentes con variaciones de $\mathrm{y}^{+}$(Makowski y Kim., 2001). Para los parámetros de turbulencia, la entrada y la salida tienen un valor de intensidad turbulenta del $5 \%$ y una relación de viscosidad turbulenta igual a 10 (García at al, 2018) Se analizaron estudios aerodinámicos previos para determinar los criterios para la selección de la velocidad de entrada. "Para un modelo digital genérico de un automóvil de NASCAR las simulaciones de CFD en 3D se realizaron a una velocidad de $50 \mathrm{~m} / \mathrm{s}(180 \mathrm{~km} / \mathrm{h})$ " (McBeath, 2015). "La velocidad del aire en la entrada se estableció uniforme a $70 \mathrm{~km} / \mathrm{h}$ " (López at al, 2016), acorde a la información obtenida, la velocidad de ingreso del aire depende de las condiciones en las cuales se desenvolverá el vehículo. 
El campo de fabricación de autopartes en el Ecuador es limitado y en algunos casos se efectúa de manera artesanal. En este contexto, este trabajo se enfoca en utilizar técnicas de ingeniería avanzada para diseñar y analizar un alerón para mejorar la eficiencia aerodinámica de un vehículo Suzuki modelo Forsa, el objetivo de este proyecto de ingeniería aplicada es desarrollar la simulación en ANSYS Fluent utilizando el modelo de turbulencia RANS k- $\epsilon$ con una malla gruesa y una malla refinada para la fabricación de un alerón de aplicación automotriz con materiales compuestos de matriz poliéster y refuerzo de fibra de vidrio.

\section{METODOLOGÍA}

El análisis del diseño aerodinámico, se sustenta en dos fases: el análisis numérico por las ecuaciones de gobierno del flujo de aire alrededor del vehículo y la simulación de dinámica de fluidos computacional (CFD). Las ecuaciones utilizadas en este trabajo fueron tomadas de McBeath, S, 2015 y Granados y González, 2017.

\section{Ecuaciones de Gobierno}

Las ecuaciones de gobierno que relacionan velocidad, presión, densidad y viscosidad de un fluido son las Ecuaciones de Navier Stokes. En el caso del flujo alrededor de un vehículo, el fluido se puede considerar incompresible y en este caso las ecuaciones de gobierno de acuerdo a la simplificación de Reynolds, en otras palabras, las ecuaciones RANS (Reynolds Averaged Navier Stokes) se definen como:

$$
\begin{aligned}
& \nabla u_{i}=0 \\
& \frac{\partial \bar{u}_{i}}{\partial t}+\bar{u}_{j} \frac{\partial \bar{u}_{i}}{\partial x_{j}}=-\frac{1}{\rho} \frac{\partial \bar{p}}{\partial x_{i}}+\frac{v \partial \bar{u}_{i}}{\partial x_{j} \partial x_{j}}-\frac{\partial \overline{u_{\imath}^{\prime} u_{j}^{\prime}}}{\partial x_{j}}
\end{aligned}
$$

Donde $\mu, p, \rho$ y $v$, representan la velocidad, densidad, presión y viscosidad cinemática del fluido respectivamente. Considerando este modelo, se realizará la simulación CFD del vehículo. Particularmente el modelo RANS k- $\epsilon$, se deriva de los modelos de viscosidad turbulenta de Prantl. El modelo k- $\epsilon$ permite modelar los complejos términos tensoriales de la energía cinética $(k)$ y de la tasa de disipación $(\epsilon)$ (Kobayashi y Kitoh, 1992).

A diferencia de las ecuaciones de Euler de flujo potencial, las Ecuaciones de Navier-Stokes, toman en cuenta los efectos viscosos que generan capas de corte y arremolinamientos en las zonas de la capa límite, lo que permite obtener una predicción adecuada del valor de las fuerzas de arrastre y de la presión generada sobre el cuerpo. Cuando un cuerpo se mueve en un fluido experimenta resistencia debido a la influencia de las fuerzas viscosas en la pared; a esta resistencia se le denomina fuerza de arrastre. El arrastre es una fuerza mecánica generada por la interacción y el contacto de un cuerpo rígido y un fluido. Los principales factores que afectan la magnitud del arrastre son la magnitud de la sección efectiva de impacto y la forma de la superficie (Granados y González, 2017). Adicionalmente, los estudios actuales de la dinámica de fluidos toman en cuenta valores adimensionales para poder escalar sus análisis. En este contexto, las ecuaciones que nos permiten calcular el coeficiente de presión $\mathrm{C}_{\mathrm{p}}$, el coeficiente de arrastre $\mathrm{C}_{\mathrm{D}}$ y la distancia adimensional a la pared $\mathrm{y}^{+}$. Estos valores adimensionales se calculan con las ecuaciones (3), (4) y (5) respectivamente. Los coeficientes de sustentación y resistencia al avance son medidas relativas del nivel de sustentación y resistencia puede generar un cuerpo. (Seward, 2014). La sustentación es una fuerza ocasionada por el fluido en dirección perpendicular a la dirección del movimiento del cuerpo. Su aplicación más importante se da en el diseño y el análisis de las alas de aeronaves o aeroplanos (Granados y González, 2017).

$$
C_{P}=\frac{p-p_{\infty}}{\frac{1}{2} \rho_{\infty} u_{\infty}^{2}}
$$

Donde $p$ es la presión estática en el punto donde el coeficiente de fricción es evaluado, $p_{\infty}$ es la presión estática en la corriente de aire, $\rho_{\infty}$, es la densidad de la corriente del fluido que pasa alrededor del vehículo y $\mu_{\infty}$ es la velocidad de la corriente de aire que pasa alrededor del fluido. Las variables que influyen al coeficiente de arrastre $\left(C_{D}\right)$ son la fuerza de arrastre $F_{D}, \rho_{\infty}, y \mu_{\infty}$ corresponden a las mismas variables descritas previamente. Finalmente $A$ corresponde a la sección frontal proyectada del vehículo.

$$
\begin{aligned}
C_{D} & =\frac{2 F_{D}}{\rho_{\infty} u_{\infty}^{2} A} \\
y^{+} & =\frac{y u_{\tau}}{v}
\end{aligned}
$$


En el cálculo de la distancia adimensional de la pared $y$ corresponde a la distancia de la pared en unidades de longitud, $\mathrm{y}^{+}$es la distancia normal a la pared y el superíndice "+", denota normalización en base a la unidad viscosa $\left(\delta_{v}=\mu_{\zeta} / v\right)$ (Guerrero at al, 2020).

\section{Digitalización 3D y modelación CAD.}

Con la ayuda del escáner 3D se genera la digitalización de la geometría del vehículo como se observa en la Figura 1. Los archivos obtenidos del proceso de digitalización en formato STL, son cargados al software CAD para el redibujo que generará el sólido del vehículo, como se muestra en la figura 1. Se toma como referencia la geometría de la compuerta posterior para el diseño la de geometría del alerón. Finalmente se diseñan 4 alerones con diferentes ángulos de incidencia, $5^{\circ}, 10^{\circ}, 15^{\circ}$ y $20^{\circ}$ para seleccionar la mejor opción luego de la simulación CFD.
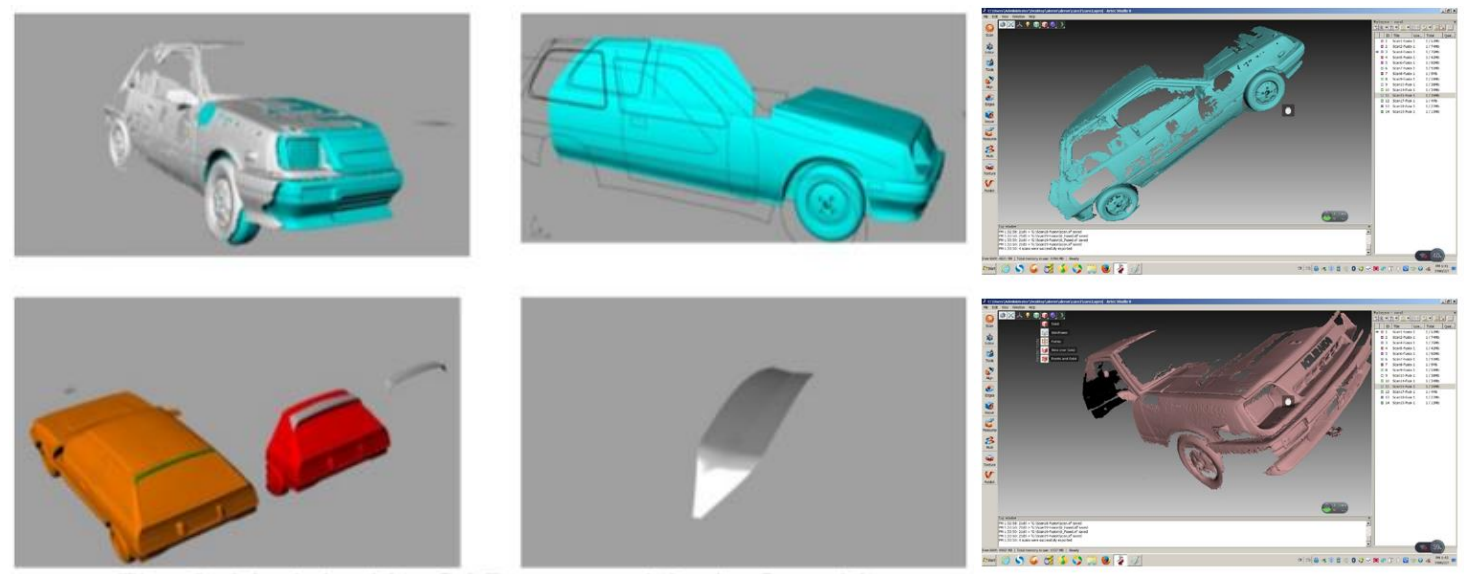

Fig. 1. Digitalización 3D y geometría del vehículo de prueba

\section{Requerimientos para modelación CFD}

Para iniciar la simulación, se debe configurar las dimensiones del dominio computacional acorde a las recomendaciones dadas por Lanfrit (2015), para ello, en función de las dimensiones del vehículo el paralepípedo de la simulación se define con las siguientes dimensiones: 3 metros de ancho, 3 metros de alto y 24 metros de profundidad, como se muestra en la Figura 2.

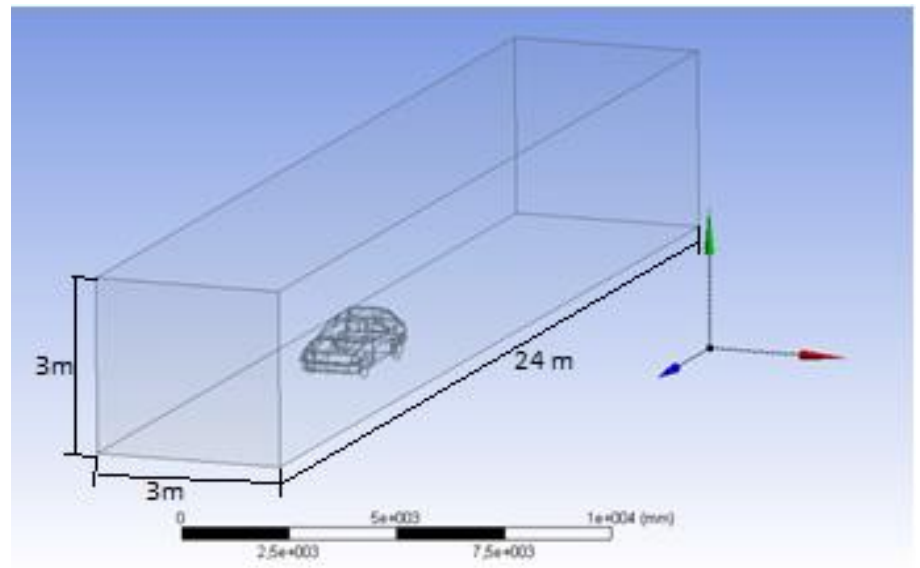

Fig. 2. Dominio computacional para simulación de vehículo Suzuki

\section{Generación de la Malla}

Se genera el mallado con la opción de tamaño, considerando proximidad y curvatura, mallado grueso, suavizado alto y tasa de incremento del $20 \%$. Sin alcanzar una buena aproximación, con un valor coeficiente de arrastre $\left(C_{D}\right)$ de 2.45, valor con un margen de error muy grande en relación a los resultados experimentales. En la Figura 3, se muestra las dimensiones del alerón en $\mathrm{mm}$, a implementar en el vehículo original. En la Tabla 1, se detalla las características del vehículo de prueba, Suzuki Forsa 1 (Adaptada de Suzuki Motor Co. Ltd., 1986). 

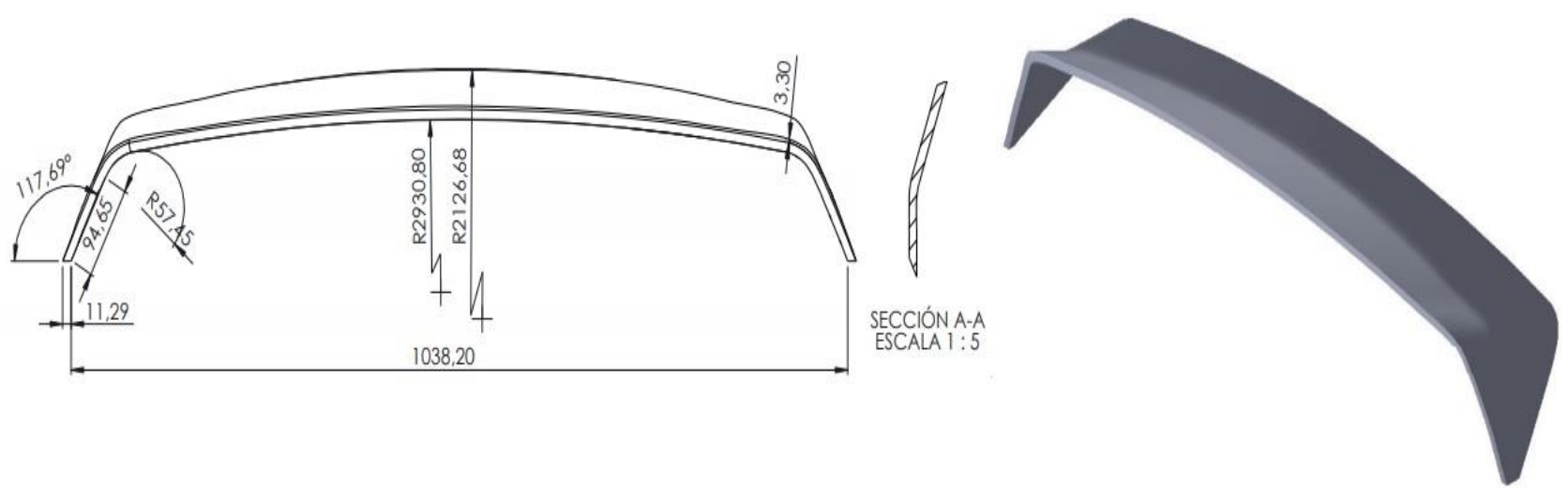

Fig. 3. Alerón implementado en el vehículo Suzuki

Tabla 1: Datos técnicos del vehículo Suzuki Forsa 1

\begin{tabular}{|l|l|}
\hline Característica & Valor \\
\hline Cilindrada (cc) & 993 \\
\hline Potencia (HP) & $48 @ 5100$ rpm \\
\hline Torque (lb-pie) & $57 @ 3200$ rpm \\
\hline Peso (kg) & 675 \\
\hline Dimensiones & Longitud:3,59 m.; Ancho:1.55 m.; Altura: $1,35 \mathrm{~m}$. \\
\hline$C_{D}$ & 0,38 \\
\hline
\end{tabular}

Para obtener resultados más precisos es fundamental refinar la calidad del mallado en la superficie del vehículo seleccionando un mallado fino con 4066365 elementos para el ajuste de la distancia de la pared adimensional a $y^{+}=5$. Para ello se debe calcular la altura de la primera celda basado en el valor objetivo que es $\mathrm{y}^{+}=5$ usando la ecuación (6), en primera instancia se obtiene el número de Reynolds de 6,6033 x 109.

$$
R e=\frac{U * L}{v}
$$

Luego se calcula con la ecuación (7), el valor del coeficiente de fricción empírico estimado en la pared para flujos externos $\left(\mathrm{C}_{\mathrm{f}}\right)$ de $639,196 \times 10^{-6}$.

$$
C_{f}=0.058 * R e^{-0.2}
$$

Con los datos obtenidos anteriormente apoyados en la ecuación (8) se calcula el esfuerzo cortante en la pared de $0,3246 \mathrm{~kg} / \mathrm{m}^{*} \mathrm{~s}^{2}$.

$$
\tau_{\omega}=\frac{1}{2} * C_{f} * \rho * U^{2}
$$

Con la ecuación (9), se calcula la velocidad friccional $\left(\mu_{\zeta}\right)$ con resultado de $0,5146 \mathrm{~m} / \mathrm{s}$.

$$
u_{\tau}=\sqrt{\frac{\tau_{\omega}}{\rho}}
$$

Despejando la ecuación (5) de la distancia adimensional de la pared $\left(\mathrm{y}^{+}\right)$se encuentra la distancia de la primera celda a la pared $(\Delta \mathrm{y})$ considerando el valor objetivo que es $\mathrm{y}^{+}=5$, dando como resultado $\Delta \mathrm{y}=$ $0,0001428 \mathrm{~m}$. Se adoptan los valores de espesor de la primera capa con el dato de altura de celda obtenido anteriormente por cálculo, la cantidad de capas prismáticas de la capa límite con 5 y el uso de la inflación por programa controlado. En la Figura 4, se visualizan la generación de capas prismáticas y la distancia de la pared adimensional al valor objetivo $\mathrm{y}^{+}=5$. 


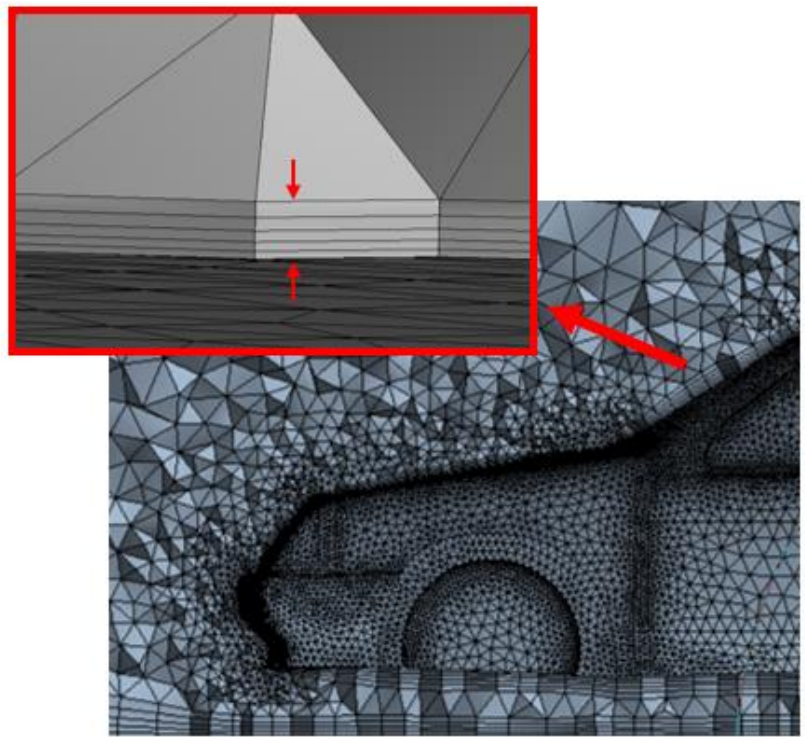

Fig. 4: Capas prismáticas y la distancia de la pared adimensional a $y^{+}=5$.

\section{Pre-procesamiento}

Se ha seleccionado el modelo de turbulencia $k-\epsilon$ con funciones de pared escalables, con los valores de $5 \%$ para la intensidad turbulenta y una tasa de 10 para la viscosidad turbulenta. Se toma como dato de la velocidad del aire $29(\mathrm{~m} / \mathrm{s})$, que es el promedio de las velocidades alcanzadas con el vehículo Suzuki Forsa en las curvas rápidas del circuito anti horario del Autódromo Internacional de Yahuarcocha.

Las ecuaciones de gobierno fueron resueltas utilizando el solver Ansys Fluent. Las ecuaciones de gobierno fueron discretizadas utilizando el método del volumen finito en estado estable. Las ecuaciones de la cantidad de movimiento y la presión fueron discretizadas utilizando esquemas de segundo orden. El algoritmo utilizado para resolver la ecuación de presión acoplada al campo de velocidad y a la ecuación de la continuidad se conoce como Coupled. A diferencia los métodos semi-implícitos y segregados como el SIMPLE, SIMPLEC y PISO, al algoritmo implícito Coupled converge más rápido y es adecuado para mallados finos o gruesos. Este método resuelve las ecuaciones de cantidad de movimiento, continuidad y presión en conjunto. El acoplamiento se alcanza a través de una discretización implícita de los gradientes de presión en las ecuaciones de Navier-Stokes (Denner, 2018).

Los valores de referencia son actualizados con la velocidad del aire y el área frontal del vehículo que se obtiene por medio de los reportes en FLUENT usando la opción proyección de área del vehículo. Los controles de solución se configuran con valores de 0,50 en los factores de relajación y los monitores de resistencia al avance (drag), con el valor - 1 en la coordenada X en el sentido del flujo del aire y el de sustentación (lift), con el valor 1 en la coordenada Z. En la primera simulación se configura la solución con 600 iteraciones para detectar el punto en cual se genera la convergencia. La convergencia llego a las 344 iteraciones en un tiempo de 10 horas utilizando un ordenador portátil con las siguientes características: Sistema operativo Windows 8 de 64 bits, procesador Intel Core 17 - $2.6 \mathrm{GHz}$, memoria RAM de 16GB, GPU gráficos GeForce GTX 860M. Se toma este valor de iteraciones como referencia para hacer las 4 series simulaciones restantes con los ángulos de inclinación de alerones propuestos.

\section{Post-procesamiento}

Con base, a los resultados de la simulación, se calcula la eficiencia aerodinámica siendo la relación entre las fuerzas sustentación negativa (carga aerodinámica) y la resistencia al avance en base a la ecuación 10 (McBeath, 2015).

$$
\text { Eficiencia aerodinamica }(\%)=\frac{\text { Fuerza carga aerodinámica }}{\text { Fuerza resistencia al avance }}
$$

Existe una relación matemática directa entre la velocidad máxima y la potencia al freno disponible, esta se denomina potencia absorbida, se calcula con la ecuación 11 (McBeath, 2015).

$$
\text { Potencia absorbida }(H P)=\frac{C_{D} \cdot A \cdot U^{3}}{1225}
$$




\section{RESULTADOS Y DISCUSIÓN}

La fuerza de resistencia al avance y la sustentación negativa de cada una de las configuraciones, se obtienen de los reportes de simulación CDF de Ansys Fluent, bajo el modelo de turbulencia k- $\epsilon$, con base en ello, se calcula la eficiencia aerodinámica y la potencia absorbida, como se muestra en la Tabla 2.

Tabla 2: Resultados simulación CFD y cálculo para las configuraciones de ángulos de alerón.

\begin{tabular}{|l|l|l|l|l|l|l|}
\hline \multicolumn{1}{|c|}{ Configuración } & Arrastre $(N)$ & $\begin{array}{c}\text { Coeficiente } \\
\text { de Arrastre }\end{array}$ & $\begin{array}{c}\text { Carga } \\
\text { Aerodinámica } \\
(N)\end{array}$ & $\begin{array}{c}\text { Coeficiente } \\
\text { Carga } \\
\text { Aerodinámica }\end{array}$ & $\begin{array}{c}\text { Eficiencia } \\
\text { Aerodinámica }\end{array}$ & $\begin{array}{c}\text { Potencia } \\
\text { Absorbida } \\
(\text { Hp) }\end{array}$ \\
\hline Suzuki Forsa Solo & 132,97 & 0,28 & $-79,84$ & 0,16 & $0,6: 1$ & 5,13 \\
\hline Suzuki Forsa con alerón 5 & 132,37 & 0,34 & $-210,10$ & 0,44 & $1,59: 1$ & 6,26 \\
\hline Suzuki Forsa con alerón 10 & 168,52 & 0,35 & $-237,64$ & 0,49 & $1,41: 1$ & 6,48 \\
\hline Suzuki Forsa con alerón 15 & 178,02 & 0,37 & $-251,82$ & 0,52 & $1,42: 1$ & 6,78 \\
\hline Suzuki Forsa con alerón 20 & 185,39 & 0,38 & $-254,63$ & 0,51 & $1,37: 1$ & 7 \\
\hline
\end{tabular}

Los valores de $\mathrm{y}^{+}=5$, están dentro de lo esperado para generar datos confiables. El ajuste de la distancia de la pared adimensional de $\mathrm{y}^{+}$es de suma importancia para obtener resultados confiables, en la Tabla 3 , se efectúan los comparativos con mallado grueso en relación al mallado fino con $\mathrm{y}^{+}=5$, se observa un margen de error en los resultados excesivamente grande sin un ajuste de la resolución de la malla.

Tabla 3: Comparativo de resultados mallado grueso versus mallado fino $\mathrm{y}^{+}=5$

\begin{tabular}{|c|c|c|c|c|}
\hline Mallado Grueso & Arrastre $(\mathrm{N})$ & $\begin{array}{l}\text { Coeficiente } \\
\text { de Arrastre }\end{array}$ & $\begin{array}{c}\text { Carga } \\
\text { Aerodinámica (N) }\end{array}$ & Coeficiente Carga Aerodinámica \\
\hline Auto con alerón $20^{\circ}$ & 1001,2 & 2,45 & $-1946,54$ & 4,77 \\
\hline Mallado Fino $Y_{+}=5$ & Arrastre $(N)$ & $\begin{array}{l}\text { Coeficiente } \\
\text { de Arrastre }\end{array}$ & $\begin{array}{c}\text { Carga } \\
\text { Aerodinámica (N) }\end{array}$ & Coeficiente Carga Aerodinámica \\
\hline Auto con alerón $20^{\circ}$ & 185,39 & 0,38 & $-254,63$ & 0,51 \\
\hline
\end{tabular}

Con base al estudio, el ángulo de incidencia de 20 grados es el más adecuado para obtener mayor carga aerodinámica según los datos obtenidos de la simulación numérica CFD por medio del método RANS k- $\epsilon$. Para la validación de los estudios CFD ejecutados, se analizan los datos del coeficiente de arrastre; respecto a ello, se puede resaltar que el valor del coeficiente arrastre para un automóvil de calle moderno es de $C_{D}=$ 0,35 (Seward, 2014), con un valor experimental del coeficiente de arrastre de $C_{D}=0,38$ (Jongeneel, 1983), se evidencia que los valores de coeficientes de arrastre obtenidos por medio de la simulación numérica acercan al valor experimental, teniendo en cuenta que el modelado CAD de la geometría compleja del vehículo fue simplificado en varias etapas hasta tener un numero aceptable de elementos que permita generar facilidades en el mallado y lograr obtener la solución minimizando los costos computacionales. El ajuste de la distancia de la pared adimensional de y+, así como el uso de un mallado fino permitió obtener resultados confiables. En base a estudios previos, tales como de Barrera (2018), se determina que el valor adimensional $\mathrm{y}^{+}=5$ es el más adecuado para obtener resultados confiables. Esto se constata en el análisis realizado con mallado grueso versus mallado fino con $\mathrm{y}^{+}=5$ en donde se observa un error importante en los valores obtenidos con el mallado grueso

En la Figura 5, se muestra la distribución de presiones en la superficie, se toma el valor de presión máxima (103.06 MPa) que se generó sobre la superficie del alerón para considéralo como dato en la simulación estructural del alerón. Las zonas de color rojo representan las máximas presiones en el vehículo, la zona verde y amarilla representan zonas de presión intermedia y las zonas de color azul representan mínima presión o no influencia de la presión. Para la manufactura del alerón se importa el diseño CAD del alerón de 20 grados al software CAM para realizar la simulación y validar que los parámetros de mecanizado sean los correctos, se generan los códigos $G$ para realizar el mecanizado del molde positivo en madera de laurel. La fabricación del alerón en matriz poliéster y fibra de vidrio se la realizó con la generación de moldes negativos en yeso a partir del molde positivo, en estos moldes negativos se colocan las capas de fibra de vidrio tipo MAT con la resina poliéster para conformar el prototipo del alerón. 


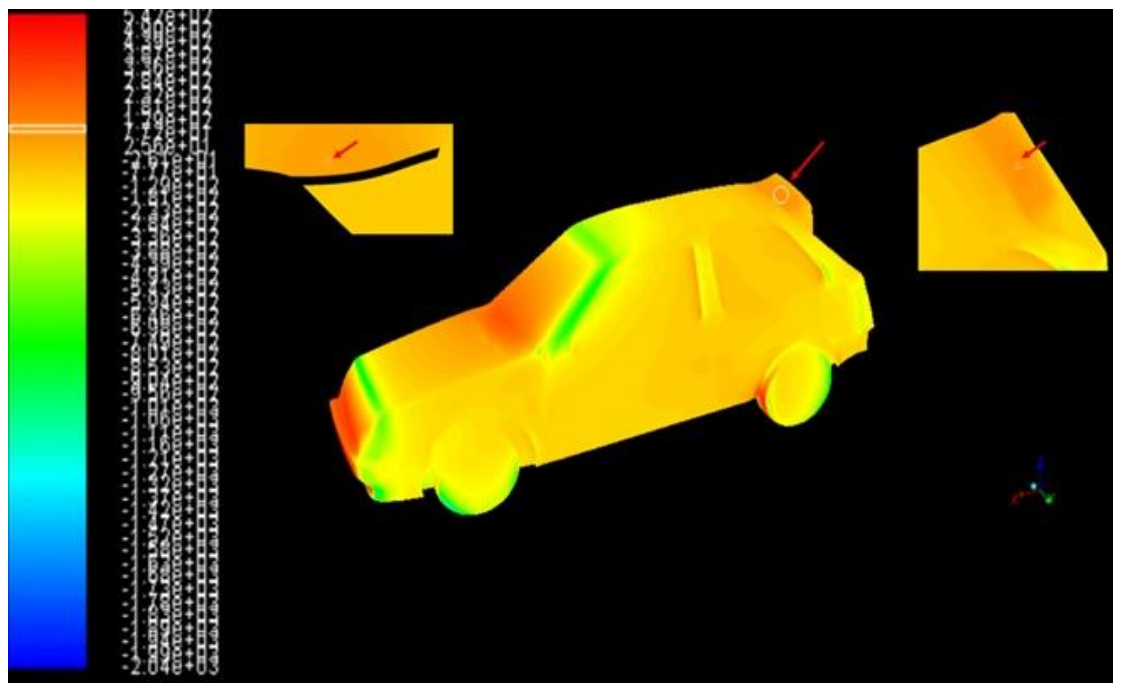

Fig. 5: Presión estática del vehículo Suzuki Forsa

\section{CONCLUSIONES}

En la presente investigación, a partir de los resultados obtenidos, se pueden extraer las siguientes conclusiones principales:

1) La configuración de alerón con ángulo de incidencia de 20 grados brinda la mayor carga aerodinámica de -254,63 N, con la mejor eficiencia aerodinámica de 1,37:1 para el vehículo Suzuki Forsa 1.

2) Para la configuración de 20 grados con 52.42 [N] de fuerza de arrastre y 1.87 [HP] de potencia absorbida, se entiende que a mayor ángulo de incidencia del alerón provoca un aumento de la fuerza de arrastre y un mayor consumo de potencia del vehículo en comparación con las otras configuraciones de ángulos estudiadas.

3) La confiabilidad de los resultados en la simulación numérica CFD, depende del ajuste correcto de la distancia de la pared adimensional de $y+$, combinado al uso de un mallado fino. Sin embargo, hay que tener presente que el modelo usado en la simulación de este estudio es un modelo más simple que el auto real, lo cual puede incidir en que las fuerzas resultantes en la práctica no sean exactamente las cargas aerodinámicas predichas en la simulación.

\section{NOTACIÓN}

$C_{D}=$ Coeficiente de arrastre;

$\mathrm{C}_{\mathrm{f}}=$ coeficiente de fricción empírico;

$\mathrm{Re}=$ Número de Reynolds;

$\mathrm{U}=$ Velocidad de flujo de aire libre, $\mathrm{m} / \mathrm{s}$;

$\mathrm{L}=$ Longitud del cuerpo, $\mathrm{m}$;

$\mathrm{v}=$ Viscosidad cinemática del aire, $\mathrm{m}^{2} / \mathrm{s}$;

$\zeta_{\omega}=$ Esfuerzo cortante en la pared, $\mathrm{kg} / \mathrm{m}^{*} \mathrm{~s}^{2}$;

$\rho=$ Densidad del aire, $\mathrm{kg} / \mathrm{m}^{3}$;

$\mu_{3}=$ Velocidad cortante de capa límite entre el fluido y la superficie del vehículo;

$\mathrm{y}^{+}=$Distancia adimensional de la pared.

\section{REFERENCIAS}

Altinisik, A., Kutukceken, E., y Umur, H., Experimental and numerical aerodynamic analysis of a passenger car: influence of the blockage ratio on drag coefficient, https://doi.org/10.1115/1.4030183, Journal of Fluids Engineering, 137(8), 081-104 (2015)

Ashton, N., y Revell, A., Comparison of RANS and DES methods for the driver automotive body, https://doi.org/10.4271/201501-1538, SAE Technical Paper, 2015-01-1538, (2015)

Barrera, E. F., Aguirre, F. A., Vargas, S., y Martínez, E. D. Influencia del y plus en el valor del esfuerzo cortante de pared a través simulaciones empleando dinámica computacional de fluidos, https://dx.doi.org/10.4067/S071807642018000400291, Información tecnológica, 29(4), 291-302 (2018) 
Chambergo, J.C., Valverde, Q., Pachas, A. A., y Yépez, H, Estudio del comportamiento fluido-dinámico de un agitador a escala reducida mediante simulación numérica, https://dx.doi.org/10.4067/S0718-07642017000300005, Información tecnológica, 28(3), 37-46 (2017)

Dhaubhadel, M. N., Review: CFD Applications in the automotive industry, https://doi.org/10.1115/1.2835492, Journal of Fluids Engineering. 118(4), 647-653 (1996)

Denner, F., Fully-coupled pressure-based algorithm for compressible flows: Linearisation and iterative solution strategies, Computers \& Fluids, vol. 175, 53-65 (2018)

Garcia, J., Acevedo, D., y otros 2 autores, D. Aerodynamic evaluation of different car carrier devices for drag reduction using CFD, https://doi.org/10.5028/jatm.v10.971, Journal of Aerospace Technology and Management, Vol.10, (2018)

Guerrero, B., Lambert, M. F., y Chin, R. Extreme wall shear stress events in turbulent pipe flows: spatial characteristics of coherent motions, Journal of Fluid Mechanics, 904 (2020)

Granados, A., y González, J., Mecánica de fluidos: teoría con aplicaciones y modelado, México: Grupo Editorial Patria, (2017)

Heinz, S., A review of hybrid RANS-LES methods for turbulent flows: Concepts and applications. https://doi.org/10.1016/j.paerosci.2019.100597, Progress in Aerospace Sciences, Volume 114, (2020)

Heft, A., Indinger, T., y Adams, N., Introduction of a new realistic generic car model for aerodynamic investigations, https://doi.org/10.4271/2012-01-0168, SAE Technical Paper, 2012-01-0168, (2012)

Hucho, W., y Sovran, G., Aerodynamics of road vehicles, https://doi.org/10.1146/annurev.fl.25.010193.002413, Annual review of fluid mechanics, 25(1), 485-537 (1993)

Jimenez, J., Computing high-Reynolds-number turbulence: will simulations ever replace experiments, https://doi.org/10.1088/1468-5248/4/1/022, Journal of Turbulence, 4 (2011)

Johansson, M., y Katz, J., Lateral aerodynamics of a generic sprint car configuration, SAE Transactions, 111, 2331-2338 (2002) Jongeneel, J., Rijden met: Suzuki SA 310 [Test Ride], Autovisie, (1983)

Katz, J., Aerodynamics of race cars, https://doi.org/10.1146/annurev.fluid.38.050304.092016, Annual Review of Fluid Mechanics, Vol 38, (2006)

Kim, S.C., y Han, S.Y., Effect of steady airflow field on drag and downforce, https://doi.org/10.1007/s12239-016-0020-2, International Journal of Automotive Technology,.17, 205-211 (2016).

Kobayashi, T., y Kitoh, K., Aerodynamics, A review of CFD methods and their application to automobile. SAE Technical Paper, 920338 (1992).

Lanfrit, M., Best Practice guidelines for handling automotive external aerodynamics with FLUENT, Fluent Deutschland GmbH, (2005)

López, O. D., Ardila, S. A., y otros 2 autores, Analysis of the vorticity in the near wake of a station wagon, https://doi.org/10.1115/1.4034523, ASME.J. Fluids Eng. February, 139(2), 021105 (2017)

Makowski, F., y Kim S. E., Advances in external-aero simulation of ground vehicles using the steady RANS equations. SAE 2000 World Congress, Detroit; SAE Technical Paper No. 2000-01-0484, (2001)

McBeath, S., Competition car aerodynamics, 3rd Edition, Dorchester: Veloce Publishing Ltd, (2015)

Seward, D., Race car design, 1 Edición, London: Editions Palgrave, (2014)

Sterken, L., Sebben, S. y Löfdahl, L., Numerical implementation of Detached-Eddy simulation on a passenger vehicle and some experimental correlation, https://doi.org/10.1115/1.4033296, ASME. J. Fluids Eng. September 2016; 138(9): 091105, (2016)

Suzuki Motor Co. Ltd., Manual de servicio SA310 Suzuki Forsa 1, Japón, (1986)

Wang, D., y Yang S. Z., Research on factors leading to variant aerodynamic drag coefficient of different cabriolets, https://doi.org/10.1007/978-981-287-978-3_40, Society of Automotive Engineers of China (SAE-China). Proceedings of SAEChina Congress 2015, 445-455 (2015)

Wang, Y., Xin, Y., y otros cuatro autores, Numerical and experimental investigations on the aerodynamic characteristic of three typical passenger vehicles. https://doi.org/10.1177/1687814018797506, Journal of Applied Fluid Mechanics, 7, 659-671 (2014) 
